\title{
Identification of Arabidopsis Loci Required for Susceptibility to the Downy Mildew Pathogen Hyaloperonospora parasitica
}

\author{
Mireille Van Damme, ${ }^{1}$ Annemiek Andel, ${ }^{1}$ Robin P. Huibers, ${ }^{1}$ Ralph Panstruga, ${ }^{2}$ Peter J. Weisbeek, ${ }^{1}$ and \\ Guido Van den Ackerveken ${ }^{1}$ \\ ${ }^{1}$ Department of Molecular and Cellular Biology, Utrecht University, Padualaan 8, $3584 \mathrm{CH}$ Utrecht, The Netherlands; ${ }^{2} \mathrm{Max}-$ \\ Planck Institute for Plant Breeding Research, Department of Plant-Microbe Interactions, 50829 Köln, Germany
}

Submitted 23 August 2004. Accepted 28 January 2005.

\begin{abstract}
Plants are susceptible to a limited number of pathogens. Most infections fail due to active defense or absence of compatibility. Many components of the plant's surveillance system and defense arsenal have been identified in the last decades. However, knowledge is limited on compatibility; in particular, the role of plant factors in the infection process. To gain insight into these processes, we have initiated an Arabidopsis thaliana mutant screen for reduced susceptibility to the downy mildew pathogen Hyaloperonospora parasitica. Ethyl methane sulfonate (EMS) mutants were generated in the highly susceptible Arabidopsis line Ler eds1-2. Eight downy mildew-resistant $(d m r)$ mutants were analyzed in detail, corresponding to six different loci. Microscopic analysis showed that, in all mutants, $H$. parasitica growth was severely reduced. Resistance of $d m r 3, d m r 4$, and $d m r 5$ was associated with constitutive expression of $P R-1$. Furthermore, $d m r 3$ and $d m r 4$, but not $d m r 5$, also were resistant to Pseudomonas syringae and Golovinomyces orontii, respectively. However, enhanced activation of plant defense was not observed in dmr1, dmr2, and dmr6. We postulate that, in these susceptibility mutants, cellular processes are disrupted which are required for $H$. parasitica infection. This interesting new set of mutants provides a basis to elucidate the molecular processes underlying susceptibility to downy mildew in Arabidopsis.
\end{abstract}

Additional keywords: disease resistance, disease susceptibility, downy mildew resistance, obligate biotroph, oomycete.

Susceptibility to plant diseases can be considered more exception than rule. Only a limited number of microbes have the capacity to retrieve nutrients and survive on living plant tissue; this capacity also is referred to as compatibility. In most cases, plants resist infection due to active defense mechanisms or the absence of compatibility toward a given pathogen. Knowledge of disease resistance has increased tremendously over the last two decades. Numerous resistance genes have been cloned and many defenseassociated and signal transduction genes have been identified (Glazebrook 2001; Rathjen and Moffett 2003). However, little is known about the molecular basis of disease susceptibility and the role of the plant in the infection process. We considered three distinct steps during infection of plants by biotrophic pathogens that involve an array of different host cell processes.

Corresponding author: G. Van den Ackerveken;

E-mail: G.vandenAckerveken@ bio.uu.nl
The first step toward compatibility is the formation of specialized penetration and feeding structures at the correct time and location. Their development requires coordinated expression of infection-related genes of the pathogen, triggered by the environment and signals from the host plant. To come into close contact with the host, some pathogens can actively move to the plant (e.g., zoospores of Phytophthora and Pythium spp. that are attracted by root exudates [Tyler 2002]). To access the host, spores attach to the plant surface, germinate, and often develop specialized structures, called appressoria, to penetrate the plant cuticle and cell wall (Tucker and Talbot 2001). Although surface hydrophobicity and hardness are sufficient to stimulate appressorium development in some pathogens (Carzaniga et al. 2001; Tucker and Talbot 2001), others require specific signals to develop infection structures (e.g., the sensing of cutin by Colletotrichum spp. [Dickman et al. 2003]), or the height of the guard cell lip by Uromyces appendiculatus (Allen et al. 1991). Also, during later stages of infection by fungal and oomycete biotrophs, signals or characteristics of the cellular environment in the host play an important role in the regulated development of haustoria and sporulation structures (Mendgen and Hahn 2002). It is striking to see that an obligate biotroph, such as the downy mildew pathogen $\mathrm{Hya}$ loperonospora parasitica, develops a single haustorium in each plant cell it encounters (Koch and Slusarenko 1990).

The second step occurs when pathogens are within the host tissue and are exposed to a wide range of activated plant-defense responses. Early pathogen detection can occur through recognition of specific elicitors, mediated by plant resistance genes (Nimchuk et al. 2003), or through perception of general elicitors (e.g., pathogen-associated molecular patterns [PAMPs] such as fungal chitin, oomycete glucans, and bacterial flagellin [Nürnberger et al. 2004]). Therefore, the circumvention of recognition and suppression of plant-defense responses is an important step for pathogens to take. For successful infection, pathogens need to reduce or modify their plant-exposed PAMPs to circumvent their recognition. This "stealth" strategy, however, does not seem to offer complete protection. Recent literature shows that pathogens have evolved advanced mechanisms to actively suppress plant-defense responses. The effector protein AvrRpt 2 of Pseudomonas syringae targets the Arabidopsis RIN4 protein, which is required for RPM1-mediated resistance (Mackey et al. 2002), resulting in RIN4 elimination (Axtell and Staskawicz 2003; Mackey et al. 2003). Another Pseudomonas effector, AvrPtoB, suppresses programmed cell death, thereby preventing the hypersensitive response (Abramovitch et al. 2003). Also, evidence is emerging in fungi 
and oomycetes that pathogen effectors exert their function on host proteins (e.g., the inhibition of the tomato pathogenesis related protease P69B by a Phytophthora infestans Kazal-type protease inhibitor [Tian et al. 2004]). Strikingly, like many bacterial effectors, fungal and oomycete effectors also appear to act inside the host cell (e.g., AvrL567 of the rust fungus Melampsora lini [Dodds et al. 2004] and ATR13 of H. parasitica [Allen et al. 2004]).

The modulation of host cell processes is an important third step in the infection process. To create a favorable environment for growth and for the acquisition of nutrients, pathogens are thought to actively influence host gene expression and metabolism. Although this seems obvious, there is, so far, little knowledge about this phenomenon. A direct link between a pathogen effector protein and host gene expression was provided by Marois and associates (2002), who showed that a set of pepper genes is induced specifically by the bacterial effector AvrBs3. In plant-bacteria interactions, the role of induced host gene expression in nutrient acquisition by the bacteria is unclear. In plant-fungus interactions, the proposed process of nutrient transport over the host cell membrane toward the pathogen is supported by the fact that sugar transporters and amino acid permeases are highly expressed in rust haustoria (Mendgen and Hahn 2002). Also, in the host plant Vicia faba, the rust fungus Uromyces fabae induces the expression of genes encoding an ATPase, amino acid transporter, asparagine synthetase, and sucrose synthase (Wirsel et al. 2001). Their contribution in providing nutrients to the intruding pathogen awaits functional analysis. In fact, no host proteins specific for the extrahaustorial membrane have been identified, so far.

The role of the host in disease susceptibility has been addressed in several recent studies. In a search for Arabidopsis loci required for susceptibility to Erysiphe cichoracearum, Vogel and Somerville (2000) screened ethyl methane sulfonate (EMS) and T-DNA insertion mutants for loss of susceptibility. Twenty-six powdery mildew-resistant ( $p m r$ ) mutants were isolated, corresponding to six loci that displayed strongly reduced sporulation, of which three have been cloned. PMR6 encodes a pectate lyase-like protein (Vogel et al. 2002), PMR4 a callose synthase (Nishimura et al. 2003), and PMR5 a protein of unknown function (Vogel et al. 2004). The PMR proteins all appear to act at the cell wall to provide resistance to powdery mildew. Natural variation in disease susceptibility of Arabidopsis to the fungal pathogen Botrytis cinerea was explored by Denby and associates (2004), who analyzed recombinant inbred lines of a Ler $\times$ Col- 0 cross. They identified several small- to medium-effect quantitative trait loci that govern disease susceptibility to Botrytis spp.

To gain more insight into disease susceptibility, we study the interaction between the oomycete downy mildew pathogen $H$. parasitica and its natural host, Arabidopsis. This pathosystem has been extensively studied over the past years and has revealed fascinating aspects of pathogen recognition, defense signal transduction, and resistance gene evolution (Holub 2001; Slusarenko and Schlaich 2003). The infection process starts with the germination of a spore and subsequent penetration of the leaf surface through the anticlinal wall of the epidermal cell layer (Koch and Slusarenko 1990). Once inside the leaf, $H$. parasitica makes intercellular hyphae from which haustoria develop in almost each adjacent plant cell. Although the pathogen breaches through the plant cell wall, it remains separated from the host cell cytoplasm by the extrahaustorial membrane. To identify host genes involved in the establishment of a successful infection, we have undertaken a genetic approach. Here, we describe the isolation and characterization of eight downy mildew-resistant $(d m r)$ mutants corresponding to six different loci. In three $d m r$ mutants, resistance was associated with constitutive activation of plant-defense responses. Three other mutants, $d m r 1, d m r 2$, and $d m r 6$, showed resistance in the absence of enhanced defense responses, suggesting that the corresponding genes are required for susceptibility to downy mildew.

\section{RESULTS}

\section{Isolation of $\boldsymbol{d m r}$ mutants.}

Mutants were created by EMS in the Landsberg erecta (Ler) mutant eds1-2 (Parker et al. 1996). EMS was chosen to find not only knock-out mutants but also missense mutations generating more subtle phenotypes. An additional advantage was that we could perform the mutagenesis in the genetic background of the eds 1-2 mutant. EDS1 (enhanced disease susceptibility 1) is a lipase-like protein (Falk et al. 1999) and is an important component in the signal transduction pathway of several resistance genes of the toll interleukin 1 receptornucleotide binding site leucine-rich repeat (TIR-NBS-LRR) class and in basal resistance to a number of pathogens (Aarts et al. 1998). Ler eds1-2 plants are highly susceptible to the downy mildew pathogen $H$. parasitica; they support more abundant sporulation of $H$. parasitica than the susceptible parental Ler line. The high level of $H$. parasitica infection of eds 1-2 facilitated the convenient distinction between phenotypes in our mutant screen for loss of susceptibility.

The $d m r$ mutants were identified among the susceptible $\mathrm{M}_{2}$ plants by visual screening of cotyledons and primary leaves for lack of $H$. parasitica sporulation at 7 to 10 days postinoculation with isolate Cala2 (Figure 1 illustrates the screening approach). From our screening of 3,600 $\mathrm{M}_{1}$ families (approximately $100,000 \mathrm{M}_{2}$ plants), we identified approximately 300 putative mutants. Of these, less than $10 \%$ could be confirmed in the next generation $\left(\mathrm{M}_{3}\right)$ as being truly resistant; the remaining $90 \%$ apparently were false positives. To rule out the possibility of pollen or seed contamination, mutants were genotyped using EDS1 primers for the parental eds 1-2 deletion. In all, 20 confirmed $d m r$ mutants were identified. The detailed analysis of eight $d m r$ mutants is described below.

\section{Genetic analysis.}

Complementation crosses between the mutants revealed six independent loci, denoted $d m r l$ to $d m r 6$. For $d m r 1$, three independent alleles were identified ( $d m r l-1$ to -3 ). To determine the chromosomal map position of the $D M R$ genes, the mutants were crossed to the FN2 Col-0 mutant. This mutant is susceptible to the $H$. parasitica isolate Cala2, due to a fast neutron mutation in the RPP2A gene (Sinapidou et al. 2004). All eight $d m r$ mutations appeared to be recessive because the $\mathrm{F}_{1}$ plants were susceptible to Cala2, and approximately a quarter of the $\mathrm{F}_{2}$ plants displayed $H$. parasitica resistance. From each cross, the resistant $\mathrm{F}_{2}$ plants were selected, genotyped, and rescreened for resistance in the $\mathrm{F}_{3}$ generation. The resulting map positions for the different $d m r$ mutations are depicted in Figure 2. The map position of $d m r l$ was determined to be below the centromere on chromosome 2 in a $1-\mathrm{Mb}$ region, between $\mathrm{BAC}$ T24I21 and T17A5. Loci $d m r 2$ and $d m r 6$ mapped between BAC MOP9 and T11H3 on chromosome 5. Although they map to the same region, $d m r 2$ and $d m r 6$ are not allelic, because $\mathrm{F}_{1}$ plants resulting from complementation crosses were susceptible to $H$. parasitica. Locus $d m r 3$ also is located on chromosome 5 , in a region flanked by marker nga151 and BAC MVA3. The $d m r 5$ mutation mapped on chromosome 3 to a 4$\mathrm{Mb}$ region, between marker $\mathrm{T} 17 \mathrm{~B} 22$ and BAC MAG2. The $d m r 4$ mutant conferred a very strong and reproducible resistance to $H$. parasitica, but could not be linked to a distinct position on the chromosomal map in a cross with Col-0 FN2, 
because the $F_{2}$ and $F_{3}$ progeny did not give a reproducible resistance phenotype.

Disease susceptibility of $\boldsymbol{d m r}$ mutants.

The $d m r$ mutants varied in degree of resistance to $H$. parasitica. To quantify the level of resistance, we counted the number of sporangiophores on mutant seedlings (Fig. 3). Compared with the parental line, Ler eds 1-2, all mutants showed strongly reduced sporulation after infection with the isolates Cala2 and Waco9. In particular, mutants $d m r 1-1$, dmr3, and $d m r 4$ showed strong to complete resistance. Mutant $d m r 5$ still supported some pathogen growth, resulting in sporulation levels that were $10 \%$ of that of the parental line. Interestingly, the different alleles of $d m r l$ showed different levels of resistance, with $d m r 1-1$ giving higher levels of resistance than $d m r 1-2$ and $d m r l-3$. In addition to their resistance to $H$. parasitica, some of the $d m r$ mutants have additional phenotypes. For example, $d m r 3$ (Fig. 4F) and $d m r 4$ (Fig. 4G) showed dwarfism, and dmrl-1 showed chlorosis and reduced growth (Fig. 4B). Except for the dwarfism in $d m r 3$ that appears to be a pleiotropic effect, the additional phenotypes did not co-segregate with resistance to $H$. parasitica in $\mathrm{F}_{2}$ mapping populations, indicating that they were caused by additional point mutations resulting from the EMS mutagenesis.

The infection process in the different $d m r$ mutants was studied microscopically to determine when and where $H$. parasitica growth and development was arrested. Infected leaves were stained with trypan blue 3 days postinoculation with Cala2 and analyzed by differential interference contrast microscopy (Fig. 4). Colonization of cotyledons and leaves of $d m r 1-1, d m r 2, d m r 3$, and $d m r 4$ by $H$. parasitica was strongly reduced compared with the parental Ler eds $1-2$ line.

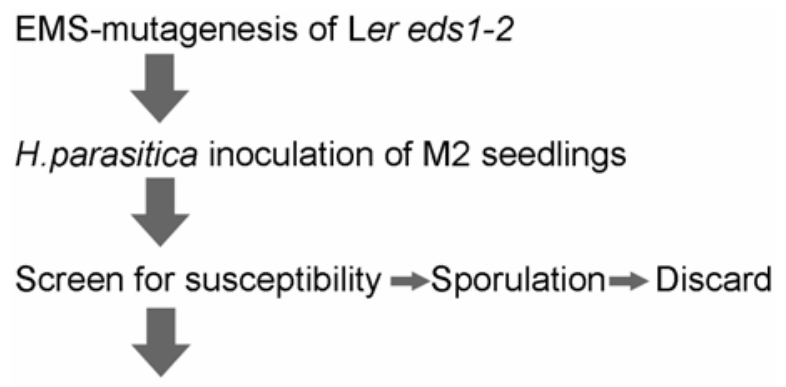

No sporulation (pursue to M3)
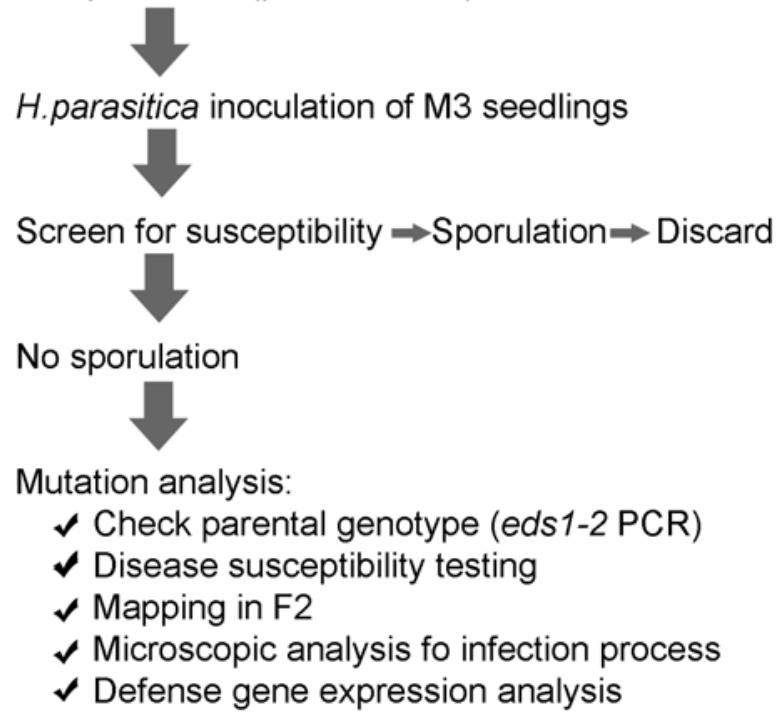

Fig. 1. Screening approach for the isolation of downy mildew-resistant mutants.
Hyphal growth still could be detected in the dmrl-2, dmrl-3, $d m r 2, d m r 5$, and $d m r 6$ mutants, although to a lesser extent than in the parental line Ler eds l-2 (Fig. 4A). In the three $d m r l$ mutants, cell wall appositions often were observed around arrested haustoria (Fig. 4B through D). These papillae, containing callose (as detected by aniline blue staining; data not shown), possibly were a secondary effect on the invading haustoria. In the case of the $d m r 2$ mutant, no papillae formation was detected; however, hyphal growth often was arrested after the first haustoria had formed (Fig. 4E). In many cases, haustoria had aberrant shapes with globular extension on the otherwise spherical haustoria, in particular in mutants $d m r 3$ (Fig. 4F) and $d m r 6$ (data not shown). On $d m r 4$ leaves, spores did not seem to be able to penetrate efficiently

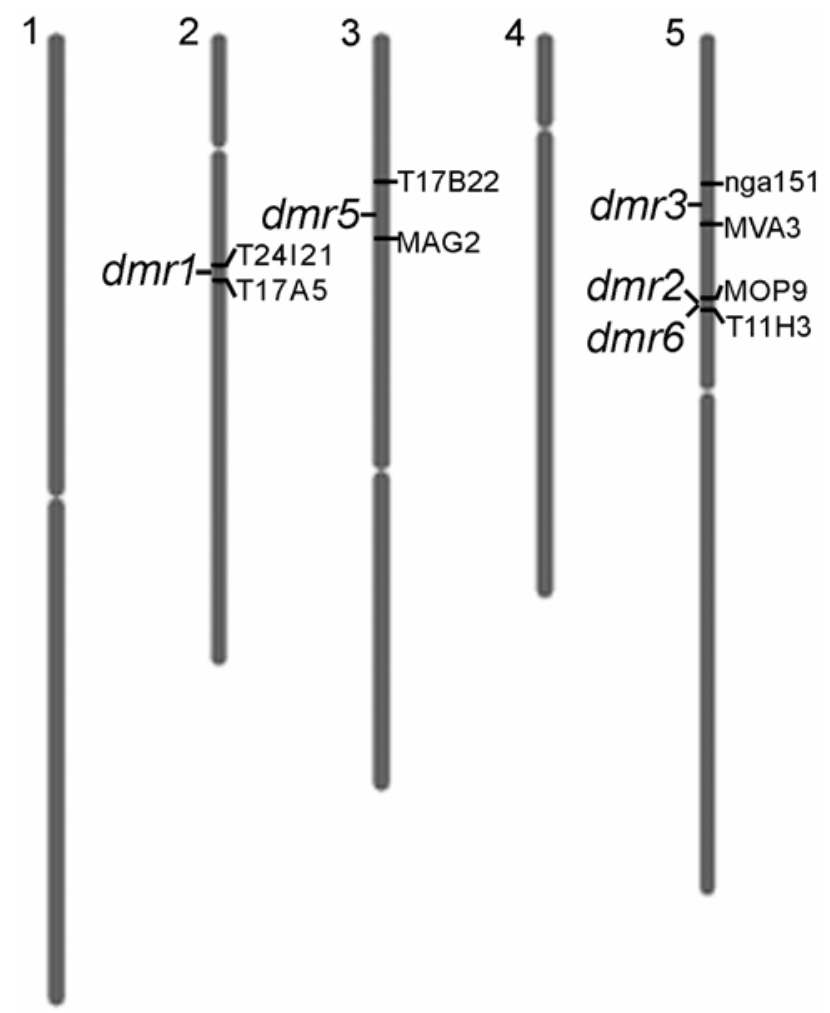

Fig. 2. Position of five downy mildew-resistant ( $d m r$ ) loci on the Arabidopsis chromosome map. The location of each $d m r$ locus is placed on the physical map using the program Chromosome Map Tool.

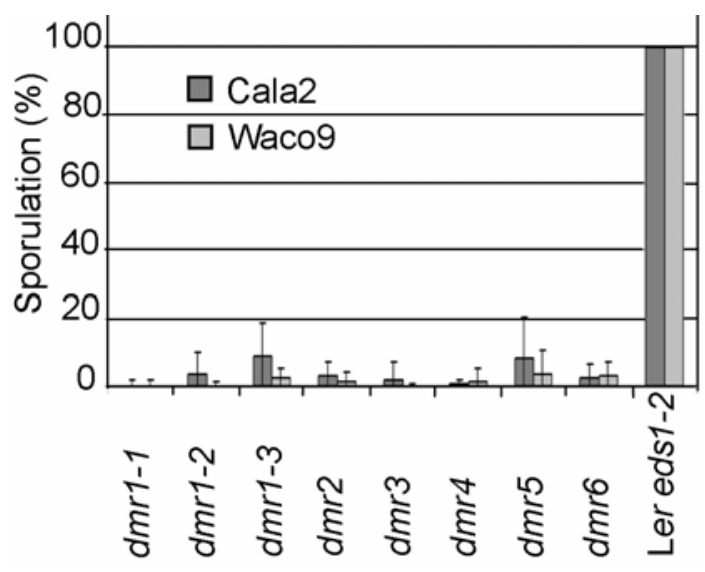

Fig. 3. Quantification of Hyaloperonospora parasitica sporulation on the downy mildew-resistant (dmr) mutants compared with the parental Ler $e d s 1-2$ line (set to $100 \%$ ) at 7 days postinoculation with the isolates Cala2 and Waco9. 
and to grow intercellular hyphae (Fig. 4G). Although there was significant $H$. parasitica colonization in $d m r 5$, haustoria were not formed in every adjacent cell and hyphal growth was irregular (Fig. 4H). In the $d m r 6$ mutants, the growth of $H$. parasitica was constrained after the first haustoria had formed (Fig. 4I). $H$. parasitica infections in the $d m r$ mutants at 5 days postinoculation (data not shown) were very similar to those at 3 days postinoculation, indicating that further colonization remained disturbed. Overall, the growth and development of $H$. parasitica, as observed by microscopic analysis of trypan blue-stained plants, was in good agreement with the level of sporulation that we have observed (Fig. 3).

The production of reactive oxygen intermediates (ROI) often is linked to the hypersensitive response and to cell wall-based
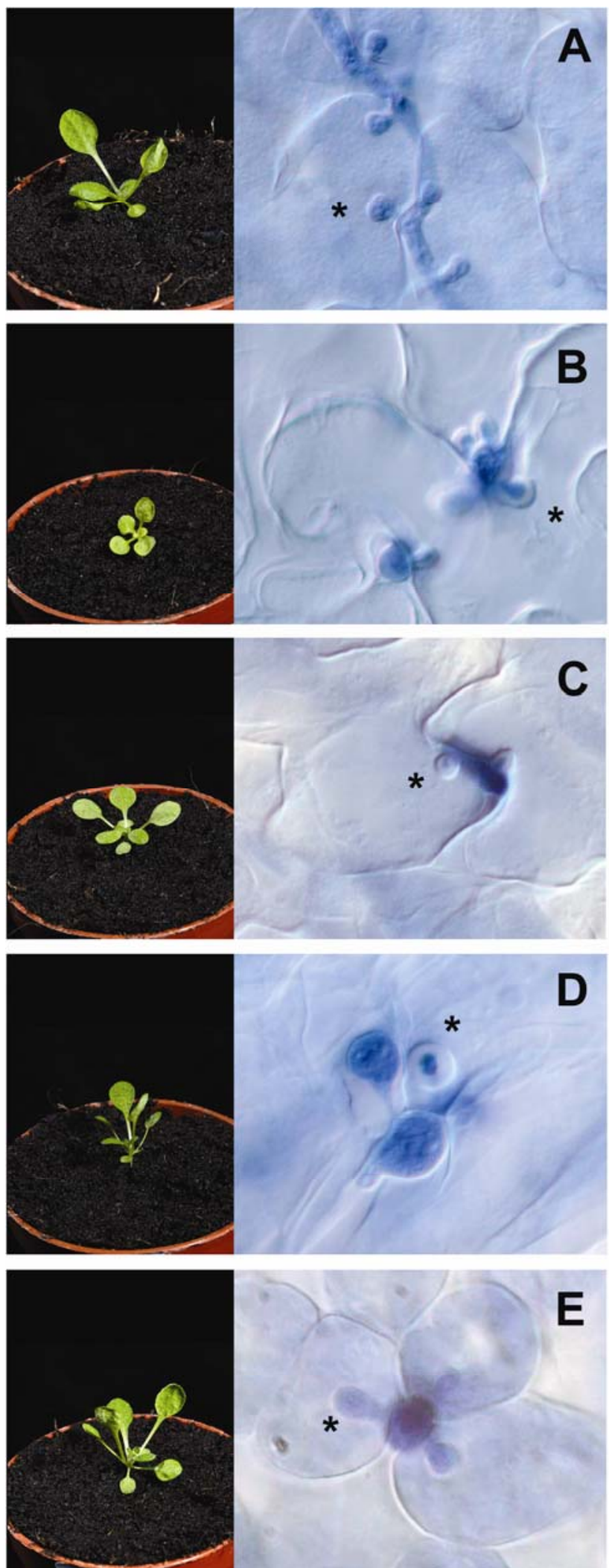

*

D
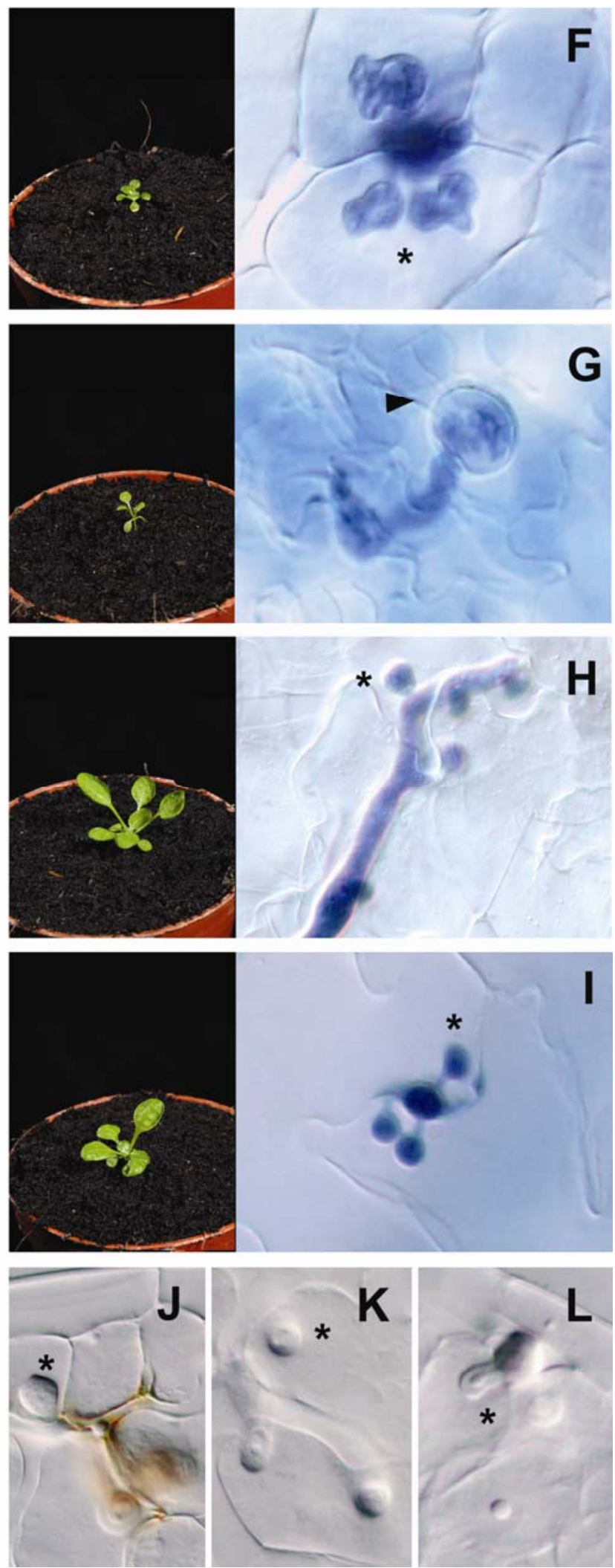
defense responses (Beers and McDowell 2001). Microscopic detection of ROI by 3'-3'-diaminobenzidine (DAB) staining showed that ROI were produced in none of the $d m r$ mutants in response to $H$. parasitica infection (Fig. 4L; data shown for dmrl-3), as is the case in the Ler eds1-2 parental line (Fig. $4 \mathrm{~K})$. As a positive control for the DAB staining, Col-0 was inoculated with the incompatible isolate Cala2. ROI could be visualized by the brown DAB precipitate that was formed in close vicinity to the invading pathogen (Fig. 4J).

To analyze whether the $d m r$ mutants displayed a spectrum of resistance broader than to $H$. parasitica alone, susceptibility to the gram-negative bacterial pathogen Pseudomonas syringae pv. tomato DC3000 and the biotrophic powdery mildew fungus Golovinomyces orontii was tested. The parental line supported growth of $P$. syringae pv. tomato (a 100-fold increase in 3 days), whereas seedlings treated with benzothiadiazole (BTH), a chemical inducer of systemic acquired resistance, showed 20-fold less bacterial growth (Fig. 5). Mutants $d m r 1, d m r 2, d m r 5$, and $d m r 6$ did not seem to be significantly affected in their susceptibility to $P$. syringae pv. tomato. In contrast, $d m r 3$ and $d m r 4$ displayed a strong resistance to $P$. syringae pv. tomato that was comparable with $\mathrm{BTH}$-treated control plants. In addition, the dmr3 and dmr4 mutants also showed resistance to the powdery mildew pathogen, G. orontii. This fungal pathogen, formerly referred to as E. orontii, previously was reported to represent one of three powdery species that are able to successfully colonize Arabidopsis thaliana (Plotnikova et al. 1998). The $d m r$ mutants were screened for resistance to $G$. orontii (Table 1). Two additional accessions, Col-0 and Shakdara (Sha), were included as susceptible and resistant controls, respectively. Like the susceptible control (Col-0, average disease resistance score 2.5), our parental line Ler-eds1-2 was susceptible (score 2.8) to powdery mildew,

Table 1. Susceptibility of the downy mildew-resistant mutants to Golovinomyces orontii

\begin{tabular}{lc}
\hline Genotype & Average disease resistance score $^{\mathbf{a}}$ \\
\hline$d m r 1-1$ & 0.5 \\
$d m r 1-2$ & 2.8 \\
$d m r 1-3$ & 2.7 \\
$d m r 2$ & 3.0 \\
$d m r 3$ & 1.2 \\
$d m r 4$ & 0.9 \\
$d m r 5$ & 2.0 \\
$d m r 6$ & 1.5 \\
Lereds $1-2$ & 2.8 \\
Col-0 & 2.5 (susceptible) \\
Sha & 0.5 (resistant) \\
\hline
\end{tabular}

${ }^{\mathrm{a}}$ Level of sporulation was scored on a disease resistance scale of 0 to 3 at 10 days postinoculation. The total number of plants tested per accession or mutant was six. The assay was repeated once with comparable results. whereas Sha proved resistant (score 0.5). The level of susceptibility of the dmrl mutants to $G$. orontii was not univocal. Whereas $d m r l-1$ was resistant (score 0.5), $d m r 1-2$ and $d m r l-3$ were equally susceptible (approximately 2.8) with the parental line. A fourth $d m r l$ mutant, $d m r l-4$, that we recently isolated was also susceptible (data not shown), suggesting that $d m r l$ does not mediate resistance to $G$. orontii. Resistance to powdery mildew in the dmrl-1 mutant could be caused by another independent mutation (e.g., the mutation that gives rise to the chlorotic growth phenotype of $d m r 1-1)$. The $d m r 3$ and $d m r 4$ mutants displayed a strong reduction in susceptibility, with $d m r 4$ showing resistance comparable with the Sha control. Also, the $d m r 5$ and $d m r 6$ mutants were more resistant to powdery mildew than the parental control, although the reduction in disease symptoms was not as strong as in the resistant control and the $d m r 3$ and $d m r 4$ plants. Interestingly, $d m r 2$ was reproducibly more susceptible to $G$. orontii than the parental line. Based on the different infection assays, we conclude that $d m r 3$ and $d m r 4$ show broad-spectrum disease resistance against oomycete, bacterial, and fungal pathogens. The other

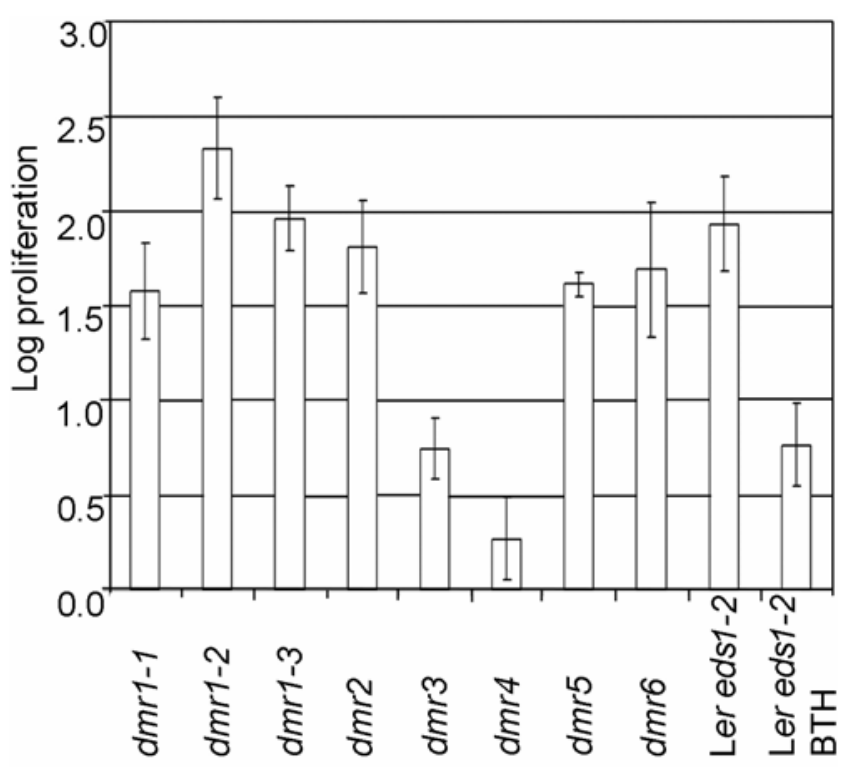

Fig. 5. Quantification of bacterial (Pseudomonas syringae pv. tomato) proliferation in the downy mildew-resistant $(d m r)$ mutants and control plants over 3 days. The $d m r 1, d m r 2, d m r 5$, and $d m r 6$ mutants showed an approximately 100-fold increase in leaf tissue comparable with that of the parental Ler eds 1-2 line. In contrast, mutants $d m r 3$ and $d m r 4$ showed a strongly reduced growth comparable with that of benzothiadiazole (BTH)treated plants ( 3 days prior to $P$. syringae pv. tomato inoculation) in which systemic acquired resistance was induced.

Fig. 4. Phenotypes of downy mildew-resistant (dmr) mutants. For each mutant, a 3-week-old plant is shown, as well as a microscopic image of Hyaloperonospora parasitica-infected tissue of 14-day-old seedlings. For each mutant, representative images of trypan blue-stained seedlings 3 days postinoculation with isolate Cala2 $\left(5 \times 10^{4}\right.$ spores $\left.\cdot \mathrm{ml}^{-1}\right)$ were taken. For clarity, a single haustorium is marked with an asterisk $(*)$ in every microscopic image. A, In the parental Ler eds 1-2 line, H. parasitica hyphal growth was abundant and haustoria were formed in adjacent plant cells. B, Mutant $d m r 1-1$ is slightly smaller and chlorotic, whereas $\mathbf{C}, d m r 1-2$ and $\mathbf{D}, d m r 1-3$ appear phenotypically similar to the parental line (A). H. parasitica growth was arrested in all three $d m r 1$ mutants and underdeveloped haustoria often were surrounded by cell wall appositions containing callose (B, C, and D). E, Infection of $d m r 2$ also showed arrested haustorial development and no additional macroscopic phenotype. F, A dwarf mutant. dmr3, showed aberrantly shaped haustoria and strongly arrested hyphal growth. G, In the dmr4 mutant, which also has a dwarf phenotype, H. parasitica penetration of the epidermal cell layer was hampered, resulting in strongly reduced intercellular growth (note the failed penetration of the germinated spore, marked with a black arrowhead). $\mathbf{H}$, The $d m r 5$ mutant shows normal plant development, but $H$. parasitica growth was reduced. There was significant hyphal growth, but haustoria were not formed in all adjacent plant cells as in the parental line (A). I, The dmr6 mutant shows slightly rounded leaves. In dmr6, some $H$. parasitica growth occurred and haustoria were formed, although they often had aberrant shapes (data not shown) or stayed immature (I). J, K, and $\mathbf{L}$, Detection of reactive oxygen intermediates (ROI) by $3^{\prime}-3^{\prime}$-diaminobenzidine staining in seedlings inoculated with $H$. parasitica isolate Cala2. J, Col-0 plants that recognize Cala2 through $R P P 2$ accumulated ROI at the infection site (visible as the red-brown precipitate), whereas $\mathbf{K}$, Ler eds 1-2 and the dmr mutants, such as $\mathbf{L}$, dmr1-3, did not show ROI production or hypersensitive cell death. 
mutants appear to be more specifically affected in their interaction with $H$. parasitica.

\section{Defense responses.}

In order to distinguish between activated defense and susceptibility mutants, the expression level of the defense-related $P R-1$ gene was determined in healthy and $H$. parasitica-inoculated seedlings of the different $d m r$ mutants. As shown by Northern blot analysis (Fig. 6), the parental line Ler-eds1-2 did not show any $P R-1$ expression, whereas BTH-treated seedlings, as expected, showed a strong induction of $P R-1$. In $d m r 3, d m r 4$, and $d m r 5$, elevated levels of $P R-1$ expression were observed. The defense gene activation in $d m r 3$ and $d m r 4$ was not unexpected because these mutants displayed resistance to $P$. syringae pv. tomato and $G$. orontii. The dmr $5 \mathrm{mu}-$ tant, however, remained susceptible to $P$. syringae pv. tomato despite elevated expression of $P R-1$. Like the parental line, mutants $d m r 1, d m r 2$, and $d m r 6$ did not show elevated $P R-1$ expression (Fig. 6). In addition, $P R-1$ expression was not induced 3 days after inoculation with the compatible $H$. parasitica isolate Cala2 (data not shown). We conclude that the mutants $d m r 1, d m r 2$, and $d m r 6$ are susceptibility mutants because they do not show broad-spectrum resistance, nor do they show enhanced defense gene expression.

\section{Is $d m r 3$ mutated in the DND1 gene?}

The $d m r 3$ mutant resembles the previously described $d n d l$ mutant in many aspects. Shared features include its map position on the upper arm of chromosome 5, resistance to both $H$. parasitica and $P$. syringae pv. tomato, constitutive expression of $P R-1$, and a dwarf phenotype. DNDI (defense no death 1) encodes a cyclic-nucleotide gated anion channel that, when mutated, leads to enhanced plant defense and constitutive pathogen resistance (Clough et al. 2000; Yu et al. 1998). To test whether $d m r 3$ is mutated in the DND1 gene, we sequenced the DNDI coding region of both the parental Ler eds 1-2 line and the $d m r 3$ mutant. No polymorphisms were detected between the mutant and parental line. This was confirmed by complementation crosses between $d m r 3$ and the Col-0 dndl mutant. The resulting $F_{2}$ and $F_{3}$ generation segregated for resistance to $H$. parasitica, indicating that $d m r 3$ and $d n d l$ are not allelic.

\section{DISCUSSION}

In this article, we describe the isolation and identification of a set of eight Arabidopsis mutants that are disturbed in the interaction with the downy mildew pathogen, $H$. parasitica. The mutant screening was based on the assumption that mutations leading to reduced pathogen growth also would result in low numbers of sporangiophores on the leaves, allowing the identification of mutants by visual screening of sporulation on $\mathrm{M}_{2}$ seedlings. Our approach is very similar to the one used by Vogel

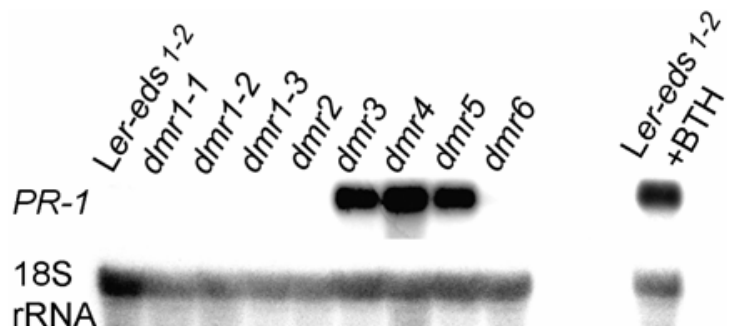

Fig. 6. Northern blot analysis of $P R-1$ expression in noninoculated seedlings of different downy mildew-resistant $(d m r)$ mutants compared with the parental Ler eds1-2 line. Ler eds1-2 treated with benzothiadiazole (BTH) was used as a positive control for $P R-1$ expression. The same membrane also was probed with $18 \mathrm{~S}$ rRNA as a loading control. and Somerville (2000) for the isolation of pmr mutants conferring resistance to powdery mildew. We expected to find two classes of downy mildew-resistant mutants, the first resulting from enhanced defense responses, and the second due to impairment of the infection process (lack of susceptibility mutants). From more than 100,000 $\mathrm{M}_{2}$ seedlings from 3,600 $\mathrm{M}_{1}$ families, we isolated 20 independent $d m r$ mutants, of which eight were analyzed in more detail. Complementation crosses showed that the eight $d m r$ mutants corresponded to six loci which subsequently were mapped in segregating $F_{2}$ populations. Two of the loci mapped to regions with known defense genes. Although $d m r 3$ mapped near $d n d l$, complementation crosses and DNDI sequencing of the $d m r 3$ mutant showed that these mutants were not affected in the same gene. On chromosome 2, dmrl mapped near sonl (Kim and Delaney 2002), which is a suppressor of niml (also known as nprl). Like $d m r 1$, sonl is a recessive mutation conferring resistance to $H$. parasitica. However, sonl mutants showed constitutive activation of $P R-1$ and resistance to Pseudomonas bacteria, whereas the three different $d m r l$ loci did not show these phenotypes. Comparison of the $d m r$ map positions to those of the $p m r$ loci (Vogel and Somerville 2000), that provide resistance to $E$. cichoracearum, indicated that they are not linked. The pmr4 locus, however, confers resistance not only to powdery mildew but also to $H$. parasitica (Vogel and Somerville 2000). PMR4 encodes a callose or glucan synthase (Jacobs et al. 2003, Nishimura et al. 2003). NahG transgenics, which do not accumulate salicylic acid (SA), appeared to be fully susceptible to E. cichoracearum (Nishimura et al. 2003). We have not identified pmr4 in our screening, either because its resistance phenotype could require $E D S 1$, or because we did not yet reach saturation in our genetic screen.

\section{Enhanced defense-response mutants.}

Many mutants already are known that show constitutive or activated plant-defense responses (e.g., the cpr, cim, acd, dnd, and $e d r$ mutants) (Glazebrook 2001). In these mutants, negative regulators of plant defense are mutated (e.g., in edrl, a MAPKKK mutant [Frye et al. 2001]) or constitutive defense activation is occurring as a result of mutated or truncated signaling proteins (e.g., acd6-1, an ankyrin repeat membrane protein [Lu et al. 2003]). Multiple mutants with constitutive defense responses form spontaneous lesions caused by cell death; these include $a c d 1, a c d 2, a c d 6, \operatorname{cpr} 5$, $l s d 1$ to 7 , and ssil (Bowling et al. 1997; Dietrich et al. 1994; Greenberg et al. 1994; Rate et al. 1999; Shah et al. 1999, Tanaka et al. 2003). In our $d m r$ mutants, no lesions or cell death was detected after $H$. parasitica inoculation. The mutant screening was performed in the eds1-2 background; therefore, we counterselected most of these mutations. Indeed several enhanced-defense mutations (i.e., cpr1 and cpr6) required a functional EDS1 gene to mediate disease resistance (Clarke et al. 2001). However, eds $1 \mathrm{mu}-$ tants still were able to mount a hypersensitive response and produce ROI (Rusterucci et al. 2001). EDS1 is an important component in the signal transduction pathway following pathogen recognition by a subgroup of resistance proteins, the TIR-NBS-LRR class (Falk et al. 1999). A second role of EDS1 is in basal pathogen resistance, explaining why eds 1 mutants are more susceptible to pathogen infection than susceptible wild-type plants (Parker et al. 1996).

Three of the $d m r$ mutants, $d m r 3, d m r 4$, and $d m r 5$, can be considered enhanced-defense mutants. Resistance of $d m r 3$ is tightly linked to dwarfism and $P R-1$ expression. This was confirmed by the analysis progeny of a backcross of $d m r 3$ to the parental line (data not shown). We have excluded the possibility that $d m r 3$ is allelic to $d n d 1$, although they map to the same chromosomal region. The map-based cloning of DMR3 will 
reveal its function in enhanced activation of plant defense. The dwarfism of $d m r 4$ is not linked to resistance to $H$. parasitica, as shown in segregating $F_{2}$ populations, and therefore probably is caused by other EMS mutations. The resistance of $d m r 4$ is particularly interesting because it appears to act at the level of penetration. Penetration resistance is a rare phenomenon but well known from powdery mildew-resistant barley mlo mutants (Panstruga and Schulze-Lefert 2002). Unlike these, however, the dmr4 mutant also showed elevated levels of $P R-1$ gene expression and resistance to a bacterial pathogen $(P$. syringae). We currently are testing whether NPRI (no PR-I expression) is required for resistance in the $d m r 4$ mutant. Although the resistance of $d m r 4$ to $H$. parasitica is strong and reproducible, it appeared not to be genetically tractable in crosses with the Arabidopsis accession Col-0. Approximately one-quarter of the $\mathrm{F}_{2}$ plants showed a clear resistance phenotype. However, $F_{3}$ plants obtained from resistant $F_{2}$ plants often proved to be susceptible. Also, several backcrosses of $F_{2}$ plants to the Col-0 mutant FN2 did not result in a reproducible phenotype. Crosses to other Arabidopsis accessions and mutants are now in progress to assess whether the $d m r 4$ phenotype is more stable in genetic backgrounds other than Col-0.

The $d m r 5$ mutant constitutively expresses the $P R-1$ gene and is resistant to $H$. parasitica but not to Pseudomonas syringae pv. tomato or $G$. orontii. We do not know of any mutant that displays this combination of phenotypes, suggesting that $d m r 5$ is affected in an as-yet-unknown component in defense signal transduction. Uncoupling of oomycete and bacterial resistance also is seen in the cpr5 nprl and cpr5 nahG double mutants, where constitutive expression of $P R-1$ and resistance to $P$. syringae pv. maculicola ES4326 is abolished, but not resistance to $H$. parasitica Noco2 (Bowling et al. 1997). The dominant disease-resistant mutant cpr6 constitutively expresses $P R-1$, $B G L-2$, and $P R-5$ and mediates resistance to $P$. syringae pv. maculicola and $H$. parasitica Noco2. Constitutive expression of $P R$ genes in the cpr6 mutant is SA but not NPR1 dependent. Resistance to $P$. syringae but not to $H$. parasitica is suppressed in the cpr6 nprl double mutant, despite $P R$ gene expression (Clarke et al. 1998). However, in addition to constitutive $P R$ gene expression, both cpr5 and cpr6 exhibit elevated levels of PDF1.2, indicating that different defense pathways are involved (Bowling et al. 1997, Clarke et al. 1998). Moreover, a functional EDS1 is fully required for resistance mediated by cpr6 and at least partially required for cpr5-mediated resistance (Clarke et al. 2001). In contrast, resistance mediated by $d m r 5$ is EDS1 independent. To unravel the role of DMR5 in plant defense, crosses to known defense signaling mutants currently are being made and defense gene expression will be monitored by DNA microarrays.

\section{Lack-of-susceptibility mutants.}

The resistance of $d m r$ mutants to $H$. parasitica also can be due to lesions in host genes that play an important role in the infection process. These so-called susceptibility mutations could well affect processes like signaling, nutrient transport, or membrane biogenesis. So far, very little is known about such host compatibility factors. Several recessive resistance loci have been described that might have arisen as a result of mutation of important "susceptibility genes" (Panstruga 2003) (e.g., barley mlo and Arabidopsis pmr) (Vogel and Somerville 2000). MLO, a plant-specific seven-transmembrane protein from barley, is required for compatibility to powdery mildew and modulates cell death and senescence (Büschges et al. 1997). Whether MLO is a true compatibility factor or a suppressor of plant defense awaits further molecular characterization of Mlo-mediated processes in the plant. The isolation of three $P M R$ genes that are required for compatibility to powdery mildew in
Arabidopsis provided an indication to their possible role in the infection process. In pmr6, a pectate lyase-like gene is mutated, resulting in an altered composition of the plant cell wall (Vogel et al. 2002), which did not block penetration by the fungus. The mechanism of pmr6-mediated resistance could not be pinpointed to a defined stage in fungal development. The powdery mildew resistance in the pmr5 mutant also is correlated to an altered cell wall composition (Vogel et al. 2004). However, currently it is not known how the change in cell wall architecture in pmr5 and pmr6 mutants translates into the posthaustorial growth cessation of the fungal pathogen. Resistance of the pmr4 mutant is associated with an enhanced pathogeninduced SA-dependent defense response that is caused by the loss of a specific callose synthase isoform (Nishimura et al. 2003).

In our screen, we have identified three candidate loci for susceptibility: $d m r 1, d m r 2$, and $d m r 6$. For $d m r 1$, three different alleles were obtained from $\mathbf{M}_{2}$ mutants of independent families and confirmed by complementation crosses. The $d m r 1, d m r 2$, and $d m r 6$ mutants showed resistance to $H$. parasitica, but not to Pseudomonas spp. or $G$. orontii. H. parasitica growth was inhibited in these $d m r$ mutants without visible cell death or accumulation of ROI. In addition, expression of the defenserelated gene $P R-1$ was not constitutive, nor more strongly expressed after inoculation with $H$. parasitica. Taken together, these data strongly suggest that cellular processes other than defense are disturbed in the $d m r l, d m r 2$, and $d m r 6$ mutants, leading to a reduced level of $H$. parasitica infection. The $d m r l$ locus was mapped on chromosome 2 to a region covering 109 genes. Both $d m r 2$ and $d m r 6$ are located near marker nga139 on chromosome 5. Genetic analysis of complementation crosses showed that $d m r 2$ and $d m r 6$ are not allelic. The $d m r 2 / d m r 6$ region still encompasses 74 genes. Fine mapping is in progress to clone the $D M R 1, D M R 2$, and DMR6 genes.

In conclusion, our genetic approach to identify Arabidopsis genes required for infection by $H$. parasitica has yielded six different mutant loci. Three of these display enhanced defense responses, which could explain their resistance phenotype. More interestingly, we have identified three new $d m r$ loci that appear to play an important role in the $H$. parasitica infection process. The isolation of the corresponding $D M R$ genes and their functional analysis will provide us with the tools to start exploring the molecular basis of susceptibility to disease, in particular downy mildew.

\section{MATERIALS AND METHODS}

Plant lines and growth conditions.

A. thaliana lines used in this study were Landsberg-erecta (Ler), Shakdara (Sha), Columbia (Col-0), Ler eds 1-2 (Parker et al. 1996), and the Col-0 mutant FN2 (Sinapidou et al. 2004). Plants were grown on potting soil in a growth chamber (Snijders Scientific, Tilburg, The Netherlands) at $22^{\circ} \mathrm{C}$ with $16 \mathrm{~h}$ of light $\left(100 \mu \mathrm{E} / \mathrm{m}^{2} / \mathrm{s}\right)$ and a relative humidity of $75 \%$.

\section{Growth and infection of downy mildew.}

H. parasitica isolate Cala2 was kindly provided by Dr. E. Holub (Warwick HRI, Wellesbourne, U.K.) and maintained on Arabidopsis Ler by weekly transfer to healthy 10- to 14-dayold seedlings (Holub et al. 1994). To obtain large amounts of sporangiospores for bioassays, inoculum was collected from Ler eds 1-2 seedlings that supported abundant Cala2 growth and sporulation (Parker et al. 1996). Inoculum $\left(5 \times 10^{4}\right.$ spores $\mathrm{ml}^{-1}$ ) was applied on 14-day-old seedlings using a spray gun. After inoculation, plants were allowed to dry for 15 to $60 \mathrm{~min}$ and subsequently incubated under a sealed lid (100\% relative humidity) in a growth chamber at $16^{\circ} \mathrm{C}$ with $9 \mathrm{~h}$ of light/day 
(100 $\left.\mu \mathrm{E} / \mathrm{m}^{2} / \mathrm{s}\right)$. The amount of sporulation was quantified at 7 days postinoculation by counting the number of sporangiophores on the cotyledons and leaves. The number of sporangiophores was determined per leaf for both isolates for at least 40 to 100 seedlings per mutant (Fig. 3). The amount of sporangiophores on Ler-eds1-2 was set as $100 \%$.

\section{EMS mutagenesis.}

Seed of Ler eds1-2 backcrossed twice to Ler were kindly provided by J. Parker (MPIZ, Köln, Germany). Approximately 10,000 seed were imbibed in water for approximately 2 days at $4^{\circ} \mathrm{C}$, then treated for $18.5 \mathrm{~h}$ in $0.2125 \%$ EMS $(21.25 \mu \mathrm{l}$ in 10 $\mathrm{ml}$ of water), and extensively washed with 2 liters of sterile water. $\mathrm{M}_{1}$ plants were grown on autoclaved soil (seven parts of potting compost and five parts of sand) and 4-week-old plants were treated with BTH (active compound at $100 \mathrm{mg} / \mathrm{liter} / \mathrm{ml}$ of $\mathrm{H}_{2} \mathrm{O}$ ) to protect them from disease. Seeds were collected as $\mathrm{M}_{2}$ families from more than 3,600 individual $\mathrm{M}_{1}$ plants. Albino mutants were detected in approximately $10 \%$ of the $\mathrm{M}_{2}$ families.

\section{Mutant screening.}

In all, 30 to $40 \mathrm{M}_{2}$ plants/family were sown in plug trays (22 by 13 wells) with fine potting soil. After 10 to 14 days, the seedlings were inoculated with $H$. parasitica Cala2. Susceptible plants were removed with tweezers 7 days later. The remaining seedlings were rescreened 3 days later and resistant plants were transferred and grown for $\mathrm{M}_{3}$ seed production. Approximately $30 \mathrm{M}_{3}$ plants per putative $\mathrm{M}_{2}$ mutant were rescreened for resistance. True resistant lines then were genotyped for the parental eds 1-2 mutation by polymerase chain reaction (PCR) to exclude seed contamination.

\section{Microscopy.}

Infections in the leaves were visualized by trypan blue staining of $H$. parasitica. For this, infected leaves were collected in a $1.5-\mathrm{ml}$ centrifuge tube. An adequate volume of lactophenol (1:1:1:1 volume of lactic acid/glycerol/phenol/ $\left.\mathrm{H}_{2} \mathrm{O}\right)$ with trypan blue $(1 \mathrm{mg} / \mathrm{ml})$ was added. The tubes were placed in a boiling water bath for $1 \mathrm{~min}$. Leaves were destained in chloral hydrate. The tubes were placed in a speed-vacuum infiltrator for $1 \mathrm{~min}$ to remove air bubbles from the leaves. $H$. parasitica growth was detected by differential interference contrast microscopy.

ROI were detected by staining for $\mathrm{H}_{2} \mathrm{O}_{2}$ accumulation. As described by Thordal-Christensen and associates (1997), DAB staining visualized $\mathrm{H}_{2} \mathrm{O}_{2}$. DAB staining was performed for $8 \mathrm{~h}$ on 10-day-old A. thaliana seedlings at 3 days postinoculation with $H$. parasitica isolate Cala2. Seedlings dissected from the root were placed in 96-well plates containing $100 \mu \mathrm{l}$ of DAB solution a plastic box under high humidity ( 5 to $6 \mathrm{~h}$ ). Afterward, the leaves were cleared of chlorophyll in alcoholic lactophenol (one volume of phenol/glycerol/lactic acid/water [1:1:1:1] and two volumes of ethanol) for 15 to $30 \mathrm{~min}$ at $65^{\circ} \mathrm{C}$, followed for $2 \mathrm{~h}$ at room temperature in fresh solution. Prior to microscopy, the samples were placed in chloral hydrate in a speed-vacuum infiltrator for $1 \mathrm{~min}$ to remove air bubbles. Arabidopsis leaves were visualized by differential interference contrast microscopy.

\section{Bacterial growth curve.}

The bacterial growth of $P$. syringae pv. tomato strain DC3000 was performed essentially as described by Tornero and Dangl (2001). Ten-day-old seedlings were inoculated by surface dipping. Samples were taken at time points 0 (1 h) and 3 days postinoculation. For this, the aerial parts of five seedlings were weighed, treated as described to release bacteria, and plated on King's B agar plates to determine bacterial numbers per milligram of fresh weight.

\section{G. orontii inoculation.}

To assess powdery mildew infection phenotypes, rosette leaves of 6-week-old $d m r$ mutants, the Ler eds 1 parental line, and two control plants (including the fully susceptible Col-0 and the resistant Sha accessions) were inoculated with a high density of $G$. orontii conidiospores. Disease symptoms were evaluated 8 to 10 days postinoculation by macroscopic and microscopic inspection and classified according to the disease resistance score described by Adam and associates (1999).

\section{Northern blot analysis.}

Total RNA was isolated from frozen tissue samples using the RNeasy plant mini kit (Qiagen, Hilden, Germany) according to the manufacturer's protocol. RNA samples were denatured for $1 \mathrm{~h}$ at $50^{\circ} \mathrm{C}$ in glyoxal $(10 \mathrm{mM}$ sodium phosphate, 1.0 M glyoxal, and $0.5 \times$ dimethyl sulfoxide). RNA samples ( 8 $\mu \mathrm{g})$ were separated on agarose $(1.5 \%)$ gel in $10 \mathrm{mM}$ sodium phosphate ( $\mathrm{pH} 7.0)$ running buffer. After transfer of the RNA to nylon membranes (Hybond N; Amersham Biosciences, Buckinghamshire, U.K.), the RNA was crosslinked to the membrane by UV-cross linking $\left(175 \mathrm{~mJ} / \mathrm{cm}^{2}\right)$. The membrane was hybridized with ${ }^{32} \mathrm{P}$-labeled probes using the random DNA labeling kit (MBI Fermentas, Vilnius, Lithuania), first with $P R-1$ and after stripping (15 min in boiling, $0.1 \times \mathrm{SSC}[1 \times \mathrm{SSC}$ is $0.15 \mathrm{M} \mathrm{NaCl}$ plus $0.015 \mathrm{M}$ sodium citrate] and $0.5 \%$ sodium dodecyl sulfate [SDS]) with $18 \mathrm{~S}$ rRNA, at $64^{\circ} \mathrm{C}$ in a hybridization buffer (7\% SDS, 0.5 M sodium phosphate, $\mathrm{pH} 7.0,10 \mathrm{mM}$ EDTA, $1 \%$ bovine serum albumen), for both probes. The $P R-1$ probe was generated by PCR amplification using the primers 5'-gtaggtgctcttgttcttcc-3' and 5'-ttcacataattcccacgagg-3'. The $18 \mathrm{~S}$ probe was derived from an Arabidopsis cDNA clone (Pruitt and Meyerowitz 1986). Filters were rinsed twice with prewarmed $\left(65^{\circ} \mathrm{C}\right)$ buffer $(0.5 \% \mathrm{SDS}, 2 \times \mathrm{SSC})$ and washed twice $15 \mathrm{~min}$ in $0.5 \% \mathrm{SDS}, 2 \times \mathrm{SSC}$ at $65^{\circ} \mathrm{C}$.

\section{Genetic mapping.}

The $d m r$ mutants were crossed to the FN2 mutant (Sinapidou et al. 2004) in the Col-0 background to generate a mapping population. $\mathrm{F}_{1}$ plants were checked for heterozygosity by genotyping with the eds1-2 PCR-based marker (forward EDS6 primer, 5'-gtggaaaccaaatttgacattag-3'; forward EDS4 primer, 5'ggcttgtattcatcttctatcc-3'; and reverse EDS2B primer, $5^{\prime}$-acacatcg gtgatgcgagaca- $\left.3^{\prime}\right)$. The $\mathrm{F}_{1}$ seedlings were drop inoculated to test for susceptibility. $F_{2}$ seedlings were screened for disease resistance as described above, and resistant $\mathrm{F}_{2}$ plants were genotyped. For fine mapping of the $d m r$ mutants, PCR-based markers were used. The markers were based on insertion or deletion polymorphisms between Colombia and Landsberg depicted on The Arabidopsis Information Resource (TAIR) website. The markers T24I21 (forward primer, 5'-aatccaaatttcttgcgagaacaca-3' and reverse primer, $5^{\prime}$-aaacgaagagtgacaatggttggag- $3^{\prime}$ ) based on a 12-bp (CER459636) deletion in the Landsberg accession. The T17A5 marker (forward primer, 5'-cgatgtctcaccggtgtaaccttta- ${ }^{\prime}$ and reverse primer, $5^{\prime}$-ttgcagagaacttcatgactggcta- $3^{\prime}$ ) resulted in a 32-bp (CER458639) deletion in Landsberg. The MOP9 marker was based on CER456575, a 31-bp deletion, in the Landsberg accession (forward primer, 5'-tttgggaacagaaaaagttggaggt-3' and reverse primer, $5^{\prime}$-catattcaaaagggaaaatcccaga- $\left.3^{\prime}\right)$. A 24-bp insertion (CER458037) in the Landsberg accession was used for the T11H3 marker (forward primer, 5'-ccaattgggttatttacttcgatt-3' and reverse primer, $5^{\prime}$-cggctttaacaacatattttcca $\left.-3^{\prime}\right)$. The nga151 marker is depicted on the TAIR website and the MVA3 marker resulted in a 16-bp insertion (CER457398) in the Landsberg accession (forward primer, 5'-cttatcgaaaccccatttgtaag- $3^{\prime}$ and reverse primer, 5'-aagaaagaggtcagagtcggagaa- $\left.3^{\prime}\right)$. The T17B22 marker resulted in a 13-bp insertion (AC012328) in the Landsberg accession (forward primer, 5' -gagattgtcttatactcggaatgtg-3' 
and reverse primer, $5^{\prime}$-ataaaatcataaccccaccaaaag- $3^{\prime}$ ) and MAG2 marker, based on a 37-bp insertion (CER464868) in the Landsberg accession (forward primer, $5^{\prime}$ - ttctattattcggtggaagatcaag- $3^{\prime}$ and reverse primer, $5^{\prime}$-tagatttctgcgaagatttct- $3^{\prime}$ ).

\section{ACKNOWLEDGMENTS}

The research of G. van den Ackerveken was made possible by a fellowship of the Royal Netherlands Academy of Arts and Sciences. We are grateful to J. Parker for providing us with BC2 Ler eds1-2 seed for EMS mutagenesis, E. Holub for the $H$. parasitica isolates and his instructions on how to work with them, J. Beynon for the FN2 seed, and A. Bent for the dnd1 mutant. We thank P. Schoondermark and B. Beusink for their efforts at the start of the mutagenesis and screening part of the project.

\section{LITERATURE CITED}

Aarts, N., Metz, M., Holub, E., Staskawicz, B. J., Daniels, M. J., and Parker, J. E. 1998. Different requirements for EDS1 and NDR1 by disease resistance genes define at least two $R$ gene-mediated signaling pathways in Arabidopsis. Proc. Natl. Acad. Sci. U.S.A. 95:10306-10311.

Abramovitch, R. B., Kim, Y. J., Chen, S., Dickman, M. B., and Martin, G. B. 2003 Pseudomonas type III effector AvrPtoB induces plant disease susceptibility by inhibition of host programmed cell death. EMBO (Eur. Mol. Biol. Organ.) J. 22:60-69.

Adam, L., Ellwood, S., Wilson, I., Saenz, G., Xiao, S., Oliver, R. P., Turner, J. G., and Somerville, S. 1999. Comparison of Erysiphe cichoracearum and E. cruciferarum and a survey of 360 Arabidopsis thaliana accessions for resistance to these two powdery mildew pathogens. Mol. Plant-Microbe Interact. 12:1031-1043.

Allen, E. A., Hazen, B. E., Hoch, H. C., Kwon, Y., Leinhos, G. M. E., Staples, R. C., Stumpf, M. A., and Terhune, B. T. 1991. Appressorium formation in response to topographical signals by 27 rust species. Phytopathology 81:323-331.

Allen, R. L., Bittner-Eddy, P. D., Grenville-Briggs, L. J., Meitz, J. C., Rehmany, A. P., Rose, L. E., and Beynon, J. L. 2004. Host-parasite coevolutionary conflict between Arabidopsis and downy mildew. Science 306:1957-1960.

Axtell, M. J., and Staskawicz, B. J. 2003. Initiation of RPS2-specified disease resistance in Arabidopsis is coupled to the AvrRpt2-directed elimination of RIN4. Cell 112:369-377.

Beers, E. P., and McDowell, J. M. 2001. Regulation and execution of programmed cell death in response to pathogens, stress and developmental cues. Curr. Opin. Plant Biol. 4:561-567.

Bowling, S. A., Clarke, J. D., Liu, Y., Klessig, D. F., and Dong, X. 1997. The cpr 5 mutant of Arabidopsis expresses both NPR1-dependent and NPR1 independent resistance. Plant Cell 9:1573-1584.

Büschges, R., Hollricher, K., Panstruga, R., Simons, G., Wolter, M., Frijters, A., van Daelen, R., van der Lee, T., Diergaarde, P., Groenendijk, J., Töpsch, S., Vos, P., Salamini, F., and Schulze-Lefert, P. 1997. The barley Mlo gene: A novel control element of plant pathogen resistance. Cell 88:695-705.

Carzaniga, R., Bowyer, P., and O'Connell, R. J. 2001. Production of extracellular matrices during development of infection structures by the downy mildew Peronospora parasitica. New Phytol. 149:83-93.

Clarke, J. D., Liu, Y., Klessig, D. F., and Dong, X. 1998. Uncoupling PR gene expression from NPR1 and bacterial resistance: Characterization of the dominant Arabidopsis cpr6-1 mutant. Plant Cell 10:557-569.

Clarke, J. D., Aarts, N., Feys, B. J., Dong, X., and Parker, J. E. 2001. Constitutive disease resistance requires EDS1 in the Arabidopsis mutants cpr1 and cpr6 and is partially EDS1-dependent in cpr5. Plant J. 26:409-420.

Clough, S. J., Fengler, K. A., Yu, I., Lippok, B., Smith, R. K., and Bent, A. F. 2000. The Arabidopsis dnd1 "defense, no death" gene encodes a mutated cyclic nucleotide-gated ion channel. Proc. Natl. Acad. Sci. U.S.A. 97:9323-9328.

Denby, K. J., Kumar, P., and Kliebenstein, D. J. 2004. Identification of Botrytis cinerea susceptibility loci in Arabidopsis thaliana. Plant J. 38:473-486.

Dickman, M. B., Ha, Y. S., Yang, Z., Adams, B., and Huang, C. 2003. A protein kinase from Colletotrichum trifolii is induced by plant cutin and is required for appressorium formation. Mol. Plant-Microbe Interact. 16:411-421.

Dietrich, R. A., Delaney, T. P., Uknes, S. J., Ward, E. R., Ryals, J. A., and Dangl, J. L. 1994. Arabidopsis mutants simulating disease resistance response. Cell 77:565-577.

Dodds. P. N., Lawrence, G. J., Catanzariti, A. M., Ayliffe, M. A., and Ellis, J. G. 2004. The Melampsora lini AvrL567 avirulence genes are expressed in haustoria and their products are recognized inside plant cells.
Plant Cell 16:755-768.

Falk, A., Feys, B. J., Frost, L. N., Jones, J. D., Daniels, M. J., and Parker, J. E. 1999. EDS1, an essential component of $R$ gene-mediated disease resistance in Arabidopsis has homology to eukaryotic lipases. Proc. Natl. Acad. Sci. U.S.A. 96:3292-3297.

Frye, C. A., Tang, D., and Innes, R. W. 2001. Negative regulation of defense responses in plants by a conserved MAPKK kinase. Proc. Natl. Acad. Sci. U.S.A. 98:373-378.

Glazebrook, J. 2001. Genes controlling expression of defense responses in Arabidopsis-2001 status. Curr. Opin. Plant Biol. 4:301-308.

Greenberg, J. T., Guo, A., Klessig, D. F., and Ausubel, F. M. 1994. Programmed cell death in plants: A pathogen-triggered response activated coordinately with multiple defense functions. Cell 77:551-563.

Holub, E. B. 2001. The arms race is ancient history in Arabidopsis, the wildflower. Nat. Rev. Genet. 2:516-527.

Holub, E. B., Beynon, J. L., and Crute, I. R. 1994. Phenotypic and genotypic characterization of interactions between isolates of Peronospora parasitica and accessions of Arabidopsis thaliana. Mol. Plant-Microbe Interact. 7:223-239.

Jacobs, A. K., Lipka, V., Burton, R. A., Panstruga, R., Strizhov, N., Schulze-Lefert, P., and Fincher, G. B. 2003. An Arabidopsis callose synthase, GSL5, is required for wound and papillary formation. Plant Cell 15:2503-2513.

Kim, H. S., and Delaney, T. P. 2002. Arabidopsis SON1 is an F-box protein that regulates a novel induced defense response independent of both salicylic acid and systemic acquired resistance. Plant Cell 14:1469-1482.

Koch, E., and Slusarenko, A. 1990. Arabidopsis is susceptible to infection by a downy mildew fungus. Plant Cell 2:437-445.

Lu, H., Rate, D. N., Song, J. T., and Greenberg, J. T. 2003. ACD6, a novel ankyrin protein, is a regulator and an effector of salicylic acid signaling in the Arabidopsis defense response. Plant Cell 15:2408-2420.

Mackey, D., Holt, B. F., Wiig, A., and Dangl, J. L. 2002. RIN4 interacts with Pseudomonas syringae type III effector molecules and is required for RPM1-mediated resistance in Arabidopsis. Cell 108:743-754.

Mackey, D., Belkhadir, Y., Alonso, J. M., Ecker, J. R., and Dangl, J. L. 2003. Arabidopsis RIN4 is a target of the type III virulence effector AvrRpt2 and modulates RPS2-mediated resistance. Cell 112:379-389.

Marois, E., Van den Ackerveken, G., and Bonas, U. 2002. The Xanthomonas type III effector protein AvrBs3 modulates plant gene expression and induces cell hypertrophy in the susceptible host. Mol. PlantMicrobe Interact. 15:637 646.

Mendgen, K., and Hahn, M. 2002. Plant infection and the establishment of fungal biotrophy. Trends Plant Sci. 7:352-356.

Nimchuk, Z., Eulgem, T., Holt, B. F., 3rd, and Dangl, J. L. 2003. Recognition and response in the plant immune system. Annu. Rev. Genet. 37:579-609.

Nishimura, M. T., Stein, M., Hou, B.-H., Vogel, J. P., Edwards, H., and Somerville, S. C. 2003. Loss of callose synthase results in salicylic acid-dependent disease resistance. Science 301:969-972.

Nürnberger, T., Brunner, F., Kemmerling, B., and Piater, L. 2004. Innate immunity in plants and animals: Striking similarities and obvious differences. Immunol. Rev. 198:249-266.

Panstruga, R. 2003. Establishing compatibility between plants and obligate biotrophic pathogens. Curr. Opin. Plant Biol. 6:320-326.

Panstruga, R., and Schulze-Lefert, P. 2002. Live and let live: Insights into powdery mildew disease and resistance. Mol. Plant Pathol. 3:495-502.

Parker, J. E., Holub, E. B., Frost, L. N., Falk, A., Gunn, N. D., and Daniels, M. J. 1996. Characterization of eds1, a mutation in Arabidopsis suppressing resistance to Peronospora parasitica specified by several different RPP genes. Plant Cell 8:2033-2046.

Plotnikova, J. M., Reuber, T. L., Ausubel, F. M., and Pfister, D. H. 1998. Powdery mildew pathogenesis of Arabidopsis thaliana. Mycologia 90:1009-1016.

Pruitt, R. E., and Meyerowitz, E. M. 1986. Characterization of the genome of Arabidopsis thaliana. J. Mol. Biol. 187:169-183.

Rate, D. N., Cuenca, J. V., Bowman, G. R., Guttman, D. S., and Greenberg, J. T. 1999. The gain-of-function Arabidopsis acd6 mutant reveals novel regulation and function of the salicylic acid signaling pathway in controlling cell death, defenses, and cell growth. Plant Cell 11:1695-1708.

Rathjen, J. P., and Moffett, P. 2003. Early signal transduction events in specific plant disease resistance. Curr. Opin. Plant Biol. 6:300-306.

Rusterucci, C., Aviv, D. H., Holt, B. F., 3rd, Dangl, J. L., and Parker, J. E. 2001. The disease resistance signaling components EDS1 and PAD4 are essential regulators of the cell death pathway controlled by LSD1 in Arabidopsis. Plant Cell 13:2211-2224.

Shah, J., Kachroo, P., and Klessig, D. F. 1999. The Arabidopsis ssil mutation restores pathogenesis-related gene expression in $n p r l$ plants and renders defensin gene expression salicylic acid dependent. Plant Cell 11:191-206. 
Sinapidou, E., Williams, K., Nott, L., Bahkt, S., Tör, M., Crute, I., BittnerEddy, P., and Beynon, J. 2004. Two TIR:NB:LRR genes are required to specify resistance to Peronospora parasitica isolate Cala2 in Arabidopsis. Plant J. 38:898-909.

Slusarenko, A. J., and Schlaich, N. L. 2003. Downy mildew of Arabidopsis thaliana caused by Hyaloperonospora parasitica (formerly Peronospora parasitica). Mol. Plant Pathol. 4:159-170.

Tanaka, R., Hirashima, M., Satoh, S., and Tanaka, A. 2003. The Arabidopsis accelerated cell death gene $A C D 1$ is involved in oxygenation of pheophorbide a: Inhibition of the pheophorbide a oxygenase activity does not lead to the "stay-green" phenotype in Arabidopsis. Plant Cell Physiol. 44:1266-1274.

Thordal-Christensen, H., Zhang, Z., Wei, Y., and Collinge, D. B. 1997. Subcellular localization of $\mathrm{H}_{2} \mathrm{O}_{2}$ in plants. $\mathrm{H}_{2} \mathrm{O}_{2}$ accumulation in papillae and hypersensitive response during the barley-powdery mildew interaction. Plant J. 11:1187-1194.

Tian, M., Huitema, E., Da Cunha, L., Torto-Alalibo, T., and Kamoun, S. 2004. A Kazal-like extracellular serine protease inhibitor from Phytophthora infestans targets the tomato pathogenesis-related protease P69B. J. Biol. Chem. 279:26370-26377.

Tornero, P., and Dangl, J. L. 2001. A high-throughput method for quantifying growth of phytopathogenic bacteria in Arabidopsis thaliana. Plant $\mathrm{J}$. 28:475-481.

Tucker, S. L., and Talbot, N. J. 2001. Surface attachment and pre-penetration stage development by plant pathogenic fungi. Annu. Rev. Phytopathol. 39:385-417.
Tyler, B. M. 2002. Molecular basis of recognition between Phytophthora pathogens and their hosts. Annu. Rev. Phytopathol. 40:137-167.

Vogel, J., and Somerville, S. 2000. Isolation and characterization of powdery mildew-resistant Arabidopsis mutants. Proc. Natl. Acad. Sci. U.S.A. 97:1897 1902.

Vogel, J. P., Raab, T. K., Schiff, C., and Somerville, S. C. 2002. PMR6, pectate lyase like gene required for powdery mildew susceptibility in Arabidopsis. Plant Cell 14:2095-2106.

Vogel, J. P., Raab, T. K., Somerville, C. R., and Somerville, S. C. 2004. Mutations in PMR5 result in powdery mildew resistance and altered cell wall composition. Plant J. 40:968-978.

Wirsel, S. G. R., Voegele, R. T., and Mendgen, K. W. 2001. Differential regulation of gene expression in the obligate biotrophic interaction of Uromyces fabae with its host Vicia faba. Mol. Plant-Microbe Interact. 14:1319-1326.

Yu, I. C., Parker, J., and Bent, A. F. 1998. Gene-for-gene disease resistance without the hypersensitive response in Arabidopsis dnd1 mutant. Proc. Natl. Acad. Sci. U.S.A. 95:7819-7824.

\section{AUTHOR-RECOMMENDED INTERNET RESOURCE}

The Arabidopsis Information Resource (TAIR) website: www.arabidopsis.org

TAIR Chromosome Map Tool:

www.arabidopsis.org/jsp/ChromosomeMap/tool.jsp 